\title{
A novel sucrose transporter gene IbSUT4 involves in plant growth and response to abiotic stress through the ABF-dependent $A B A$ signaling pathway in Sweetpotato
}

\author{
Dandan Wang ${ }^{1 \dagger}$, Hongjuan Liu ${ }^{1+}$, Hongxia Wang ${ }^{2}$, Peng Zhang ${ }^{2}$ and Chunyu Shi ${ }^{1 *}$
}

\begin{abstract}
Background: To maintain sweetpotato (Ipomoea batatas (L.) Lam) growth and yield, sucrose must be transported from the leaves to the roots. Sucrose transporters or carriers (SUTs or SUCS) transport sucrose and are involved in plant growth and response to abiotic stress. However, the mechanisms of SUTs in sweetpotato abiotic stress resistance remains to be determined.

Results: In the present study, we cloned a novel IbSUT4 gene; the protein encoded by this gene is localized in the tonoplast and plasma membrane. The plant growth was promoted in the IbSUT4 transgenic Arabidopsis thaliana lines, with increased expression of AtFT, a regulator of flowering time in plants. Over-expression of IbSUT4 in Arabidopsis thaliana resulted in higher sucrose content in the roots and lower sucrose content in the leaves, as compared to the wild-type (WT) plants, leading to improved stress tolerance during seedling growth. Moreover, we systematically analyzed the mechanisms of IbSUT4 in response to abiotic stress. The results suggest that the ABREmotif was localized in the IbSUT4 promoter region, and the expression of the ABA signaling pathway genes (i.e., ABF2, ABF4, SnRK2.2, SnRK2.3, and PYL8/RCAR3) were induced, and the expression of ABl1 was inhibited.

Conclusions: Our dates provide evidence that IbSUT4 is not only involved in plant growth but also is an important positive regulator in plant stress tolerance through the ABF-dependent ABA signaling pathway.
\end{abstract}

Keywords: Sweetpotato, Sucrose transporters, Plant growth, Abiotic stress, ABA signaling pathway

\section{Background}

In higher plants, photoassimilates are transported from source organs to sink organs mainly in the form of sucrose. The synthesis, storage, transportation, and utilization of sucrose are some of the major determinants of the cell activity, organ and tissue development, and crop yield [1]. Sucrose transportation is mediated by various proteins, of

\footnotetext{
* Correspondence: scyu@sdau.edu.cn

Dandan Wang and Hongjuan Liu both authors equally contributed to the publication

${ }^{1}$ State Key Laboratory of Crop Biology, College of Agronomic Science,

Shandong Agricultural University, Tai' an 271018, China

Full list of author information is available at the end of the article
}

which sucrose transporters or carriers (called SUTs or SUCs) mediate sucrose movement across membranes.

Plant SUTs belong to the major facilitator superfamily (MFS) and are integral membrane proteins with 12 transmembrane-spanning regions [2]. SUTs are assumed to be $\mathrm{H}^{+}$-sucrose transporters involved in cellular protoncoupled sucrose uptake and may serve two main functions in the apoplastic pathway: loading sucrose into the phloem in source leaves and unloading sucrose into the cells of sink organs, such as the storage roots, tubers, fruits, and developing tissues [3]. Several SUTs have been identified and characterized in plants. For example, Riesmeier et al.

C C The Author(s). 2020 Open Access This article is licensed under a Creative Commons Attribution 4.0 International License, which permits use, sharing, adaptation, distribution and reproduction in any medium or format, as long as you give appropriate credit to the original author(s) and the source, provide a link to the Creative Commons licence, and indicate if changes were made. The images or other third party material in this article are included in the article's Creative Commons licence, unless indicated otherwise in a credit line to the material. If material is not included in the article's Creative Commons licence and your intended use is not permitted by statutory regulation or exceeds the permitted use, you will need to obtain permission directly from the copyright holder. To view a copy of this licence, visit http://creativecommons.org/licenses/by/4.0/ The Creative Commons Public Domain Dedication waiver (http://creativecommons.org/publicdomain/zero/1.0/) applies to the data made available in this article, unless otherwise stated in a credit line to the data. 
(1992) first cloned spinach SUT cDNA and verified its transport function in yeast [4]. Subsequently, the SUTs of Arabidopsis thaliana were reported. Sauer and Stolz (1994) used spinach SUT cDNA as a probe to screen the Arabidopsis thaliana cDNA library, isolating SUT1 and SUT2 [5]. The AtSUCs were analyzed and found to consist of three distinct subfamilies. The low substrate specificity protein encoded by AtSUC2 is highly expressed in the collection phloem, and is involved in phloem loading and the retrieval of leaked sucrose in the transport phloem [6,7]. In the absence of sucrose, AtSUC2 mutant seedlings were smaller than wild-type (WT) seedlings [8]. Compared to other SUCs, AtSUC9 has an ultrahigh affinity for sucrose. Plants with AtSUC9 mutations are sensitive to low sucrose levels and have an early flowering phenotype under shortday conditions, suggesting that AtSUC9 is associated with floral induction [9]. In contrast to AtSUC9, AtSUC4, which belongs to the SUT4 clade, has low sucrose affinity and is the only Arabidopsis SUC known to be localized in the tonoplast, releasing sucrose from the vacuole [10]. In addition to Arabidopsis thaliana, SUCs in other plants have also been studied. Inhibition of the StSUT4, mainly expressed in sink organs such as tubers, sink leaves, and flowers, leads to early flowering, increased tuber yield, and decreased in sensitivity toward a far-red light, which may be owing to increased sucrose export from the leaves at the end of the light period [11]. Transgenic poplars with RNAi-suppressed PtaSUT4 had increased leaf-to-stem biomass ratios, elevated sucrose in the source leaves and stems, and altered carbohydrate-active enzymes [12]. Together, these studies demonstrate that SUTs are involved in the carbohydrate partitioning of source and sink organs.

SUTs are also involved in the plant's response to various abiotic stressors; environmental factors impact the source-sink relationship, carbon allocation, and plant growth [1]. The loss-of-function mutation in AtSUC2 and AtSUC4 leads to hypersensitivity to drought, salt, and cold stress, while abscisic acid (ABA) treatment during seed germination and seedling growth lead to high sucrose content in the shoots but low sucrose content in the roots [13]. Compared to WT Populus plants, in those silenced for SUT4, acute drought conditions resulted in reduced water uptake rates and delayed wilting [14]. A recent global analysis of publicly available gene expression data was conducted to assess the regulation of SUT genes in response to different environmental stimuli [15]. The results indicated that SUTs respond to abiotic stressors, but the stress-induced effects on the sucrose distribution and the underlying molecular mechanisms remain unexplored.

$\mathrm{ABA}$ is a broad-spectrum phytohormone involved in the regulation of stomatal opening, growth and development, and coordination of various stress signal transduction pathways during abiotic stress [16, 17]. To date, several ABA-pathway genes responsible for plant tolerance to abiotic stress have been identified, including the ABA receptor PYL8/RCAR3 and the regulatory factors SNF1 (SNF1-activated protein kinase) and $\mathrm{PP} 2 \mathrm{C}$ ( $2 \mathrm{C}$ protein phosphatases) [18-21]. Moreover, it has been reported that ABA is associated with the sugar response pathway in plants [22]. Jia et al. (2015) found that low sucrose levels induced AtSUC9, which accumulates ABA through the ABA-pathway ABAinducible genes, to enhance abiotic stress resistance [23].

Sweetpotato is a dicotyledonous crop, with underground storage organs. Its yield is closely related to the transport and distribution of photosynthetic products. The sweetpotato SUT genes IbSUT1 and IbSUT2 were previously isolated, and an immunolocalization study found that IbSUT2 is localized in the SE-CCs (sieve element-companion cells) [24, 25]. However, the characteristics and functions of the sweetpotato SUTs and the mechanisms by which they mediate sucrose transport to support the growth and development of sink organs in response to environmental stimuli are unknown. Here, we report the double-membrane localization of a novel sweetpotato SUT gene, IbSUT4, and demonstrate its involvement in the abiotic stress response through the ABF-dependent ABA signaling pathway.

\section{Results}

\section{IbSUT4 encodes a conserved SUT protein and is} homologous to Solanum StSUT4

To investigate the functions of the sweetpotato SUTs, we cloned a gene (Gene Bank: MN233360) and named it IbSUT4. The expected 463 bp EST fragment was amplified using degenerate primers SpSUT4-F and SpSUT4-R (Fig. S1A). The specific primers were designed according to the obtained EST sequences from the amplification of $3^{\prime}$ and $5^{\prime}$ cDNA, and the lengths of the $3^{\prime}$ and $5^{\prime}$ ends of IbSUT4 were obtained (Fig. S1B). The primers were generated by splicing the sequences, and the entire $1623 \mathrm{bp}$ sequence was obtained, with the longest open reading from (ORF) of $1512 \mathrm{bp}$.

Quantitative reverse transcription PCR (qRT-PCR) indicated that IbSUT4 was expressed at very high levels throughout the plant, with relatively higher levels in the sink organs (Fig. S1C). Protein prediction software revealed that the IbSUT4 protein was 502 amino acids long and contained one MFS domain and 12 transmembrane regions, which is a typical feature of MFS family (Fig. 1a). The MFS family as a membrane transport protein, whose basic function is to assist transmembrane transport [26].

To explore the relationship between the IbSUT4 protein and other plant SUTs, phylogenetic analyses were performed with the amino acid sequences. BlastP and phylogenetic analyses showed that the protein encoded 


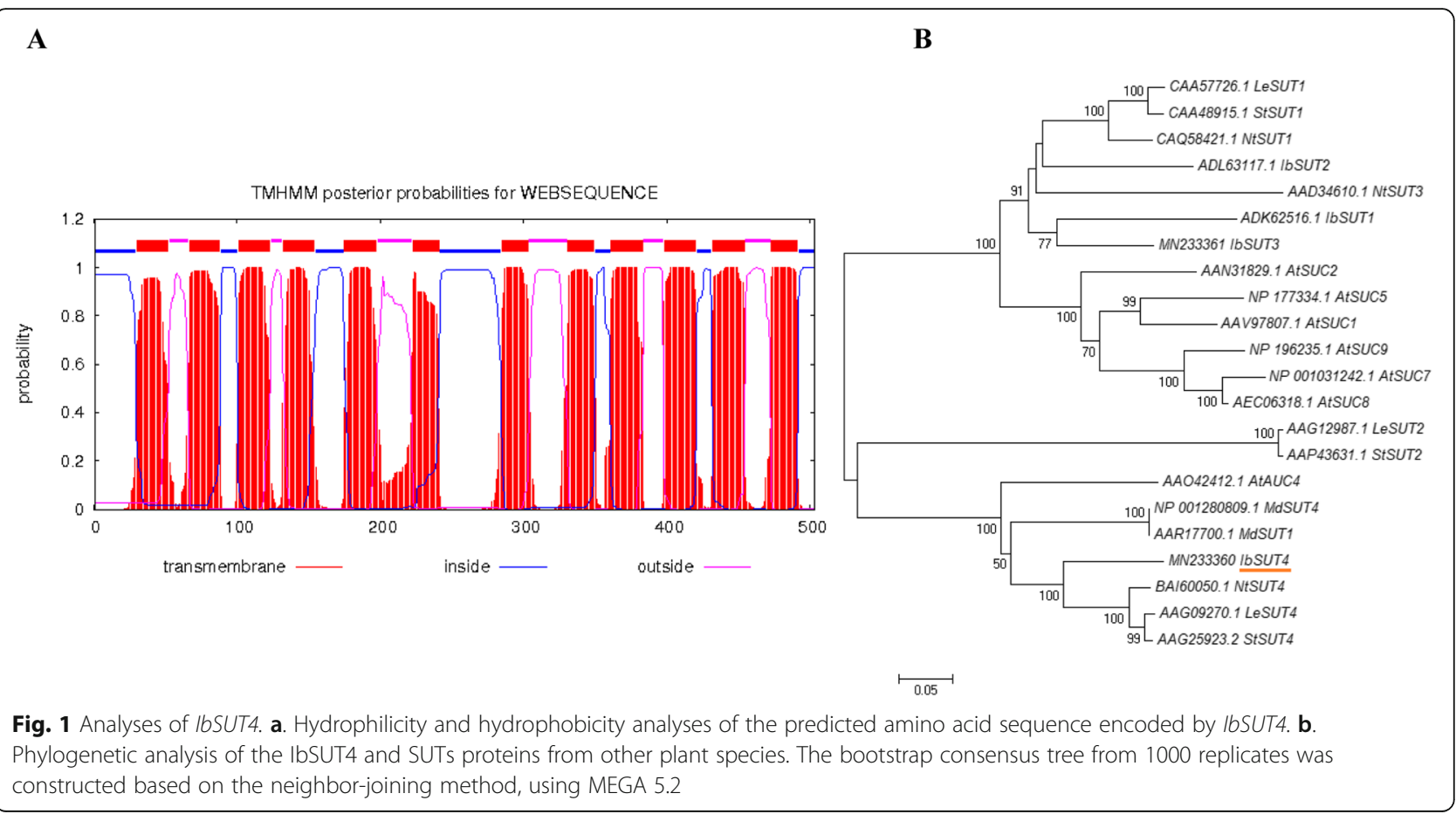

by IbSUT4 belongs to the MFS family. Furthermore, IbSUT4 belonged to clade IV and was a close homolog of StSUT4 and AtSUT4, both of which are involved in the abiotic stress response (Fig. 1b). This indicates that IbSUT4 may also play an important role in the response to abiotic stress.

\section{IbSUT4 localizes to the tonoplast and plasma membrane}

Based on the subcellular localization of StSUT4 [11, 27], we predicted that IbSUT4 would also be localized in the tonoplast and plasma membrane. We constructed a Cterminal translational fusion of GFP to IbSUT4, under the control of the Cauliflower Mosaic Virus 35S promoter (CaMV35S:IbSUT4-GFP). The GFP signal of the fusion protein was unevenly distributed along the membrane in tobacco protoplasts (Fig. 2). The irregular and rounded distribution of GFP suggested that IbSUT4 may be associated with the vacuolar membrane of tobacco protoplasts.

To test if IbSUT4 was localized in the tonoplast, we used Propidium Iodide (PI) to dye the nucleus. The nucleus was stained red, and the GFP signal was detected around the nucleus (Fig. 2). These results suggest that IbSUT4 is localized to the tonoplast and plasma membrane.

\section{IbSUT4 encodes a functional sucrose transporter}

To verify the sucrose uptake activity of the sweetpotato SUT, we diluted the yeast solution and coated the plate. On media containing $2 \%$ sucrose as the sole carbon source, SUSY7/ura3 transformed with IbSUT4 grew better than yeast transformed with an empty p416GPD vector (Fig. 3a). However, on media containing $2 \%$ glucose as the sole carbon source, SUSY7/ura3 transformed with IbSUT4 and the empty p416GPD vector did not differ (Fig. 3b). The expression of IbSUT4 allowed yeast to grow on sucrose, indicating that IbSUT4 encodes a functional SUT.

To observe the activity of IbSUT4 in vivo, an expression vector 35S:IbSUT4 was constructed and introduced into Arabidopsis, resulting in six homozygous transgenic lines, resistant to hygromycin. Semi-quantitative RT-PCRs of the transcript levels of IbSUT4 in the transgenic lines showed that IbSUT4 was up-regulated in Arabidopsis thaliana (Fig. 3c). We selected SUT4-1, SUT4-7, and SUT4-10 for further analyses. We used esculin (6, 7-dihydroxycoumarin $\beta$ D-glucoside), the fluorescent sucrose analog of sucrose, to observe the transcription activity in Arabidopsis thaliana. WT and the three transgenic lines were grown on $1 / 2 \mathrm{MS}$ medium for 7 days. The plants were then incubated for $90 \mathrm{~min}$ in a $1 / 2 \mathrm{MS}$ liquid solution containing $1 \mathrm{mM}$ esculin, and the blue fluorescent signal intensity was observed. The signal intensity in the roots of transgenic Arabidopsis thaliana was significantly stronger than in WT, indicating the intracellular absorption and accumulation of esculin in the roots (Fig. 3d). To increase the accuracy and reliability of the results, the fluorescence intensity was quantified in Image $\mathrm{J}$, and the results reconfirmed previous observations (Fig. S2), implying that 


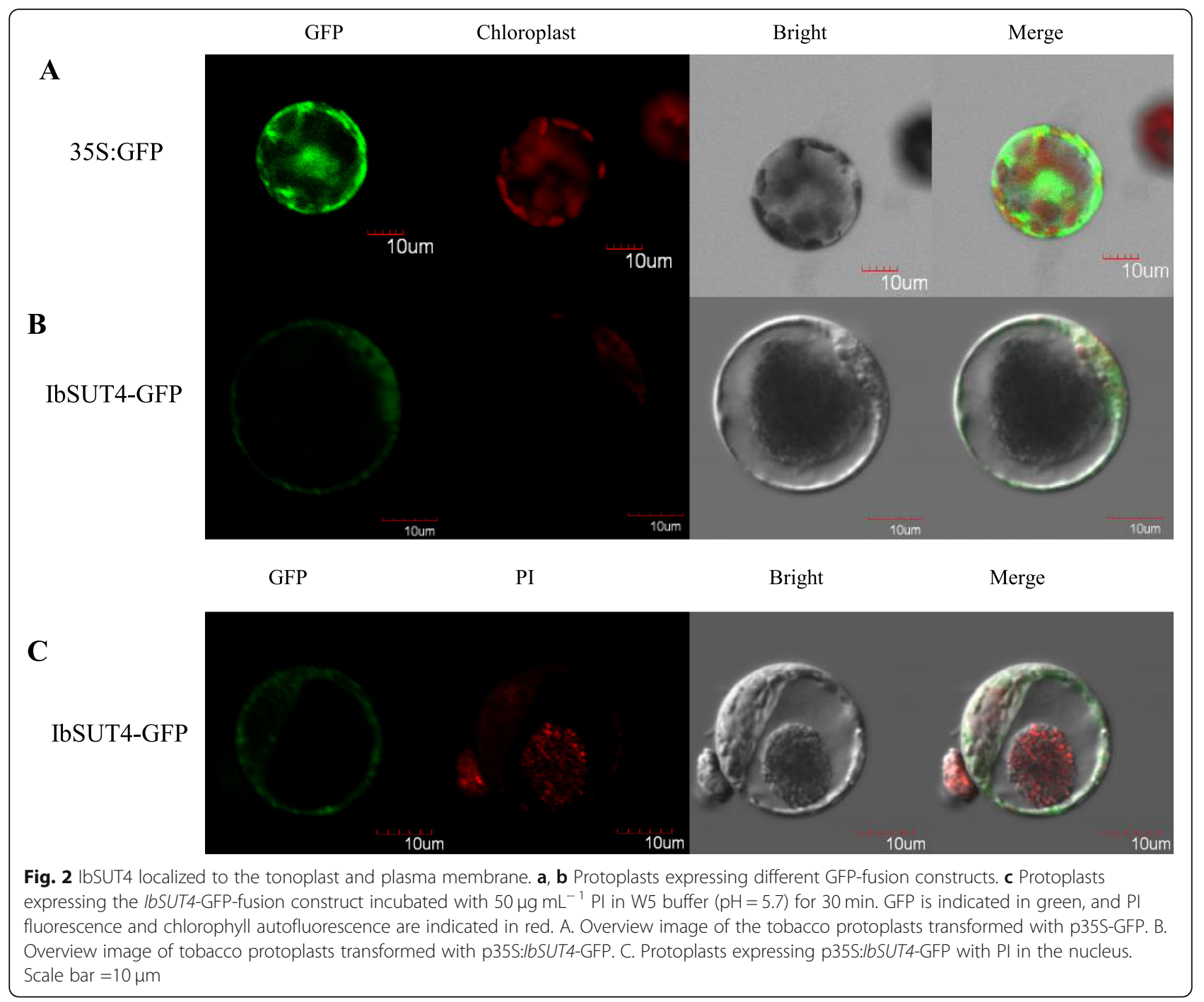

IbSUT4 showed sucrose uptake activity, and this activity was enhanced in IbSUT4-expressing lines.

\section{Over-expression of IbSUT4 results in early flowering in Arabidopsis thaliana}

After 13 days of growth under $16 \mathrm{~h}$ light / $8 \mathrm{~h}$ dark (long day, LD) conditions, we assessed the number of flowering plants (Table S2) and found that the IbSUT4-expressing Arabidopsis thaliana flowered earlier and had larger leaves than the WT plants (Fig. 4a). We also quantified the expression of the flowering marker gene, AtFT. The FLOWERING LOCUS T (FT) gene is the key locus determining the flowering time of plants through photoperiodic pathway. The $F T$ gene also plays a role in the regulation of vegetative growth and morphogenesis in plant organs [28]. The expression of AtFT differed significantly between the WT and three transgenic Arabidopsis thaliana lines (Fig. 4b), indicating that IbSUT4 expression induced early flowering. These results suggest that IbSUT4 is involved in plant growth.

\section{Over-expression of IbSUT4 improves sensitivity to ABA during seed germination and increases stress resistance during seedling growth}

To gain insights into the possible functions of IbSUT4, abiotic stress (low temperature, mannitol, and high salt) and ABA treatments were performed in sweetpotato. The qRT-PCR results suggested that IbSUT4 responds to multiple stressors (Fig. S3), based on these results, we selected high salt, low temperature, and ABA stress to investigate IbSUT4 expression in Arabidopsis thaliana.

Under control conditions, the rate of seed germination rates in the WT and transgenic lines did not differ. Under low temperature and high salt stress conditions, the germination rates were also similar. Interestingly, the germination rates of the transgenic lines were significantly 


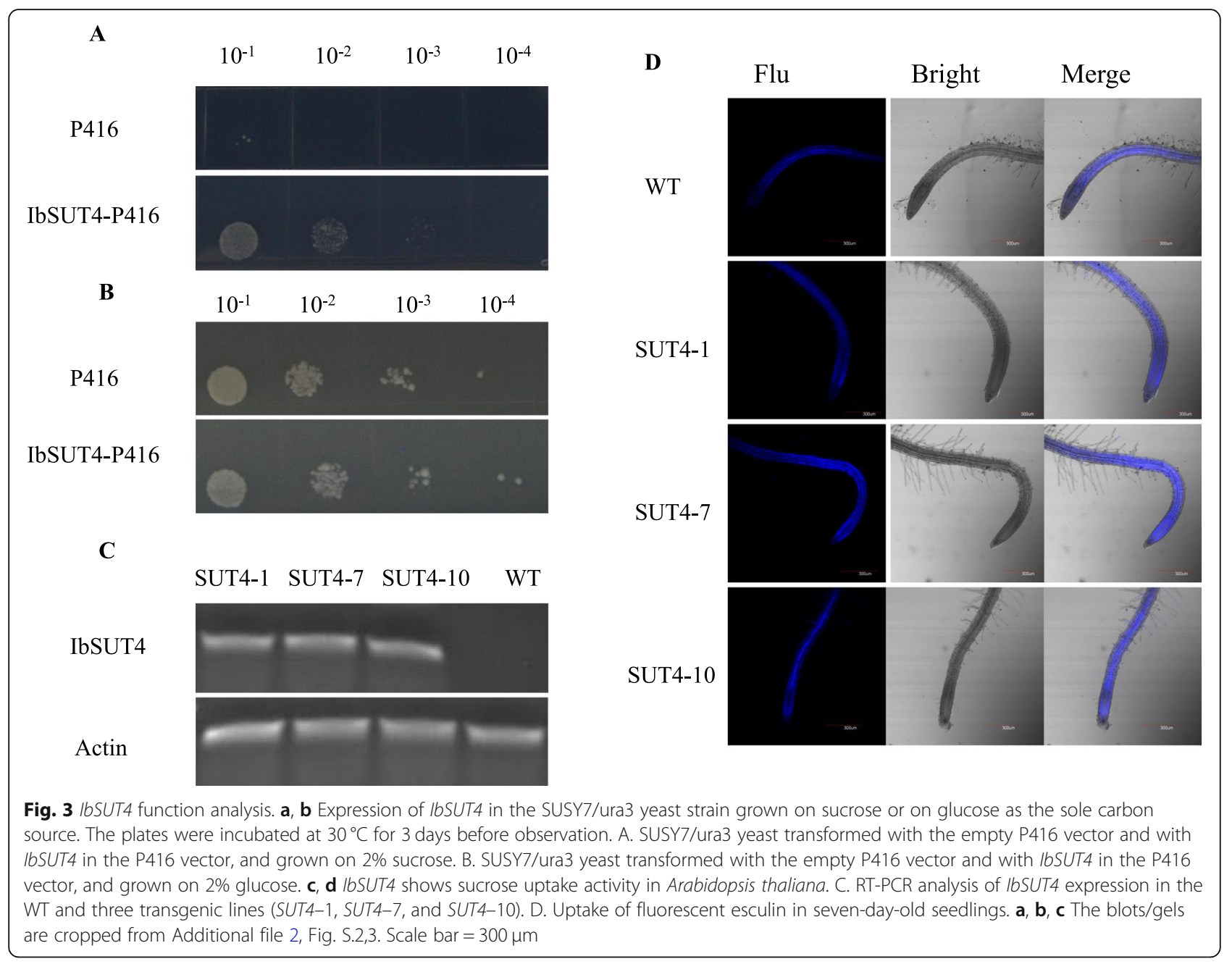

A

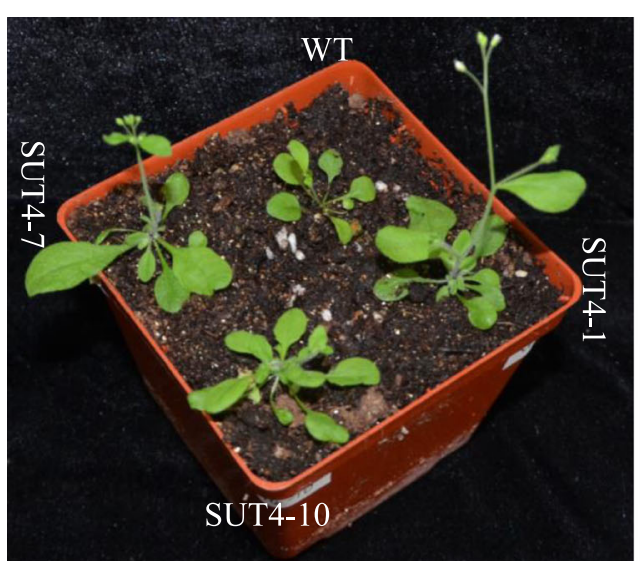

B

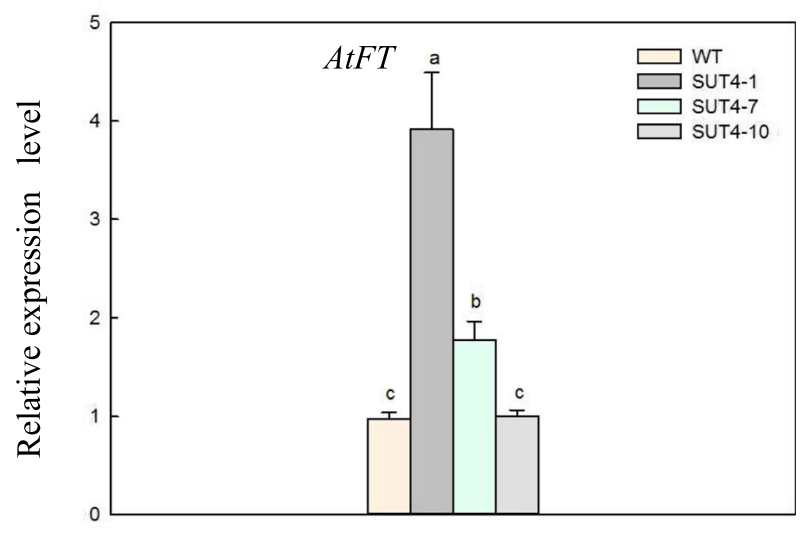

Fig. 4 IbSUT4 is involved in the plant growth. a. The three IbSUT4 transgenic lines (SUT4-1, SUT4-7, and SUT4-10) showed earlier flowering than the WT when grown under LD conditions. $\mathbf{b}$. The expression of the early flowering marker gene AtFT1. Lowercase letters indicate statistically significant differences $(P \leq 0.05)$ 
suppressed by treatment with $1 \mu \mathrm{M}$ ABA (Fig. 5a). To further investigate this phenomenon, we analyzed the AtABI3 gene by qRT-PCR. ABA and GA hormones play an important role in dormancy and germination [29, 30], and AtABI3, an ABA response factor, has a negative regulatory role in seed germination that can inhibit seed germination at high expression levels [31]. AtABI3 expression was significantly higher in the transgenic lines than in WT under $1 \mu \mathrm{M}$ ABA stress (Fig. 5b), suggesting that over-expression of IbSUT4 resulted in improved sensitivity to exogenous ABA during seed germination.

We further investigated the effects of abiotic stress on seedling growth by examining the roots lengths. There were no significant differences in roots growth between WT and transgenic lines under control conditions, but the transgenic seedlings exhibited longer roots under abiotic stress (Fig. 5c). Quantitative analyses showed that the roots of the transgenic seedlings produced less malondialdehye (MDA) (Fig. 5d). These results suggest that over-expressing of IbSUT4 can improve plant resistance to abiotic stress.

Over-expression of IbSUT4 alters the sucrose distribution in shoots and roots in response to salt, low temperature, and exogenous ABA treatments in Arabidopsis thaliana Previous studies have shown that AtSUC4 mutants exhibit altered sucrose distributions in the shoots and roots during stress [13]. Therefore, we measured the sucrose content of WT and transgenic lines under stress conditions. The roots of the transgenic lines exhibited greater accumulation of sucrose than the leaves (Fig. 6), suggesting that IbSUT4 is required for sucrose distribution to the roots under stress conditions. Interestingly, the sucrose contents of the WT and transgenic roots was higher under various stress conditions than under the control traetment, suggesting that sucrose accumulation in the roots may be important for plant stress tolerance. These results show that the sucrose distribution is altered in transgenic
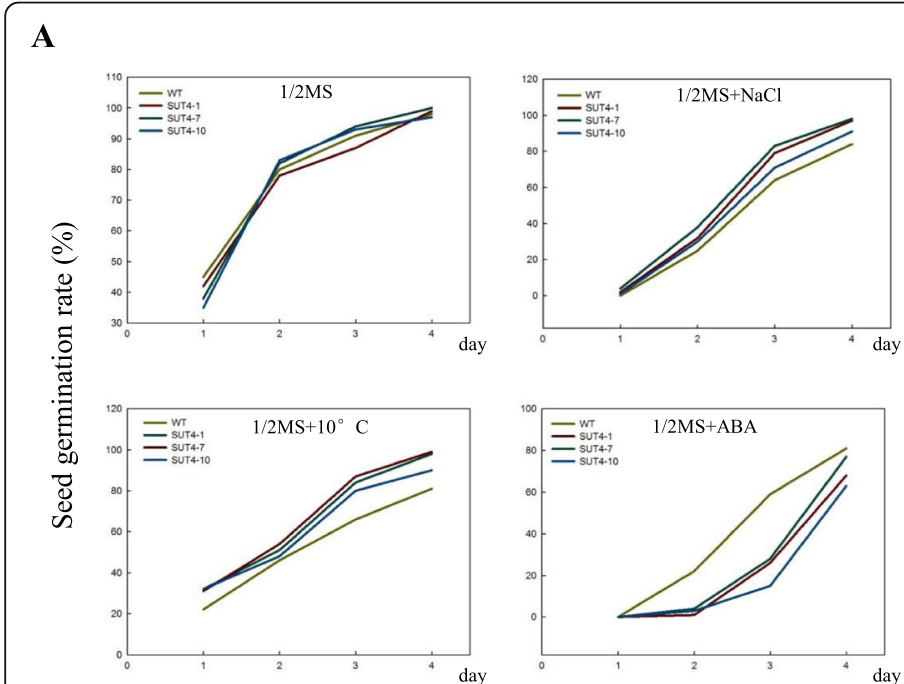

B

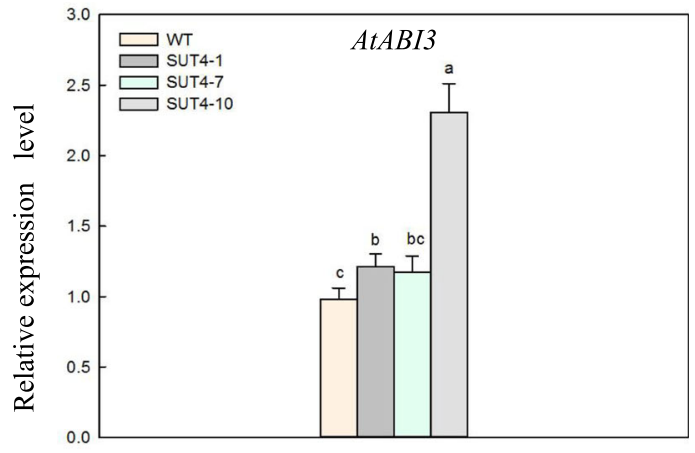

C
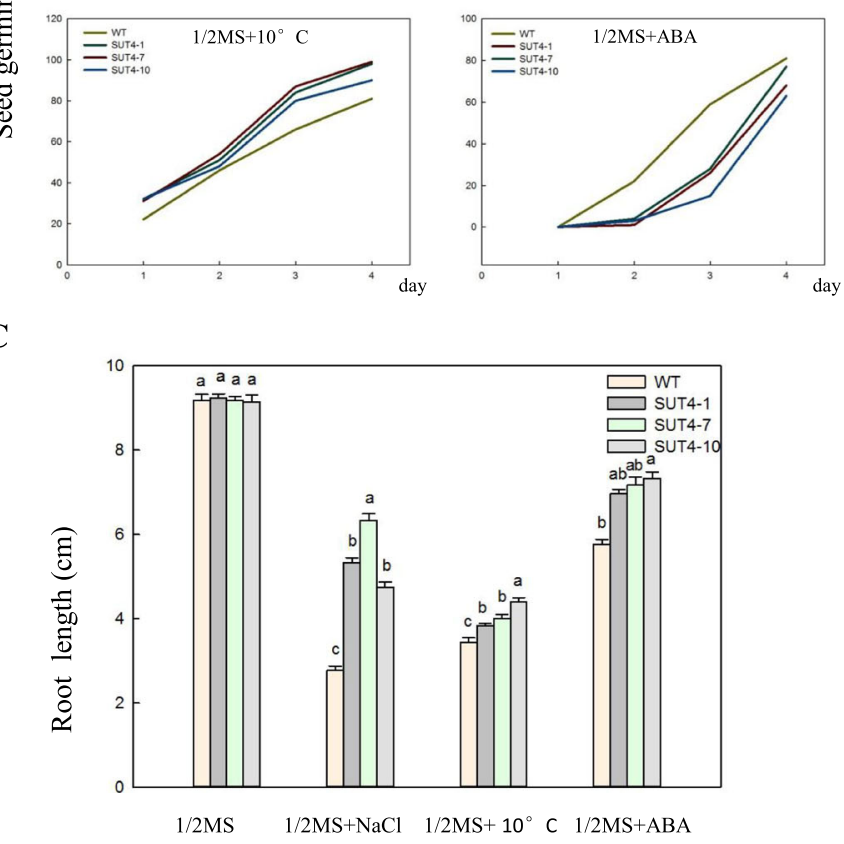

D

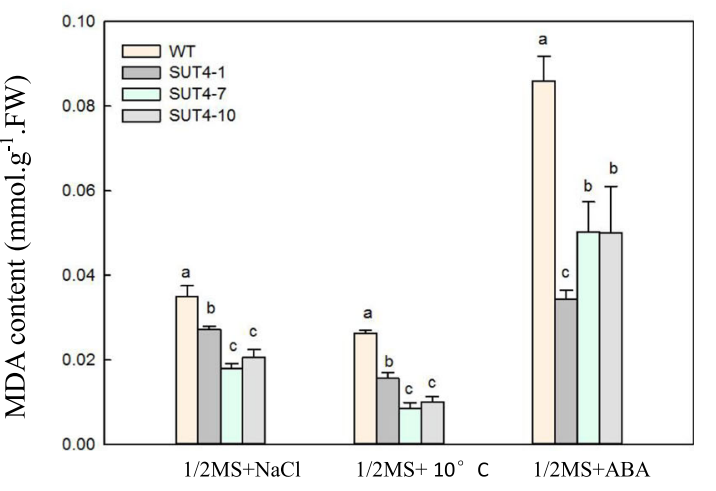

Fig. 5 Seed germination and seedling growth in the WT and the three IbSUT4 transgenic lines (SUT4-1, SUT4-7, and SUT4-10) under abiotic stress conditions. a. The germination rates were recorded in $1 / 2 \mathrm{MS}$ media with or without $\mathrm{NaCl}, 10^{\circ} \mathrm{C}$, or $\mathrm{ABA}$ treatments during a period of 1 to $4 \mathrm{~d}$ after stratification. $\mathbf{b}$. The expression of $A t A B / 3$ in seeds under the ABA treatment for $4 \mathrm{~d}$. $\mathbf{c}$ and $\mathbf{d}$. 14 day-old seedlings grown with or without $\mathrm{NaCl}, 10^{\circ} \mathrm{C}$, or $\mathrm{ABA}$ were analyzed for their root length and MDA content. Lowercase letters indicate statistically significant differences $(P \leq 0.05)$ 
A

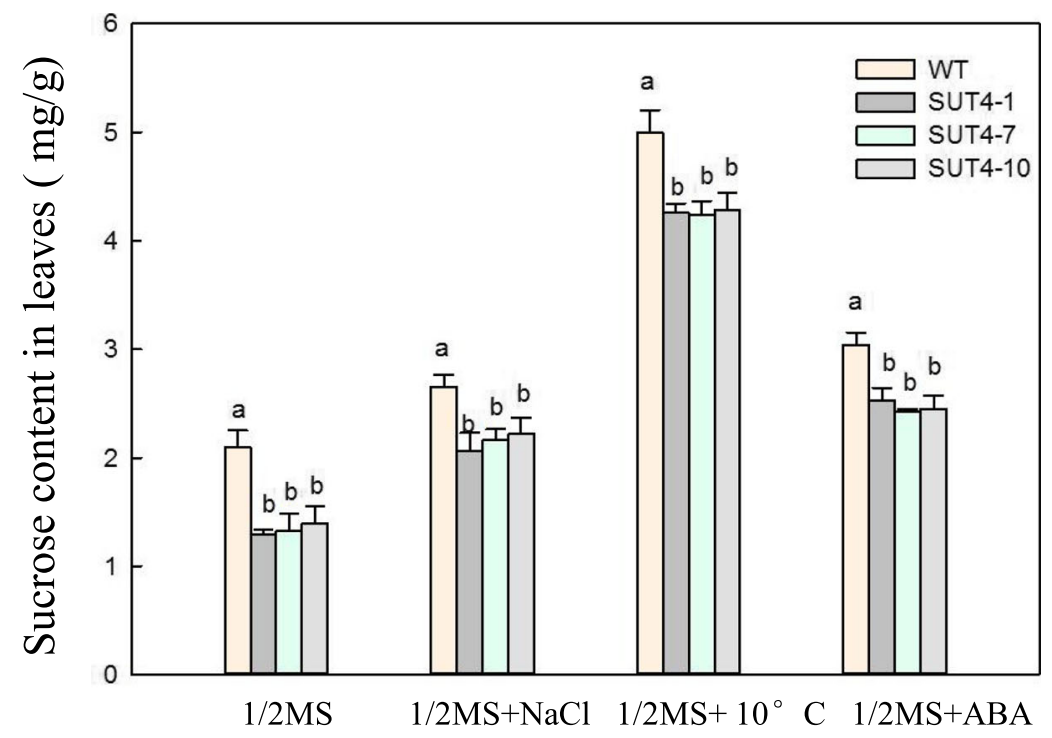

B

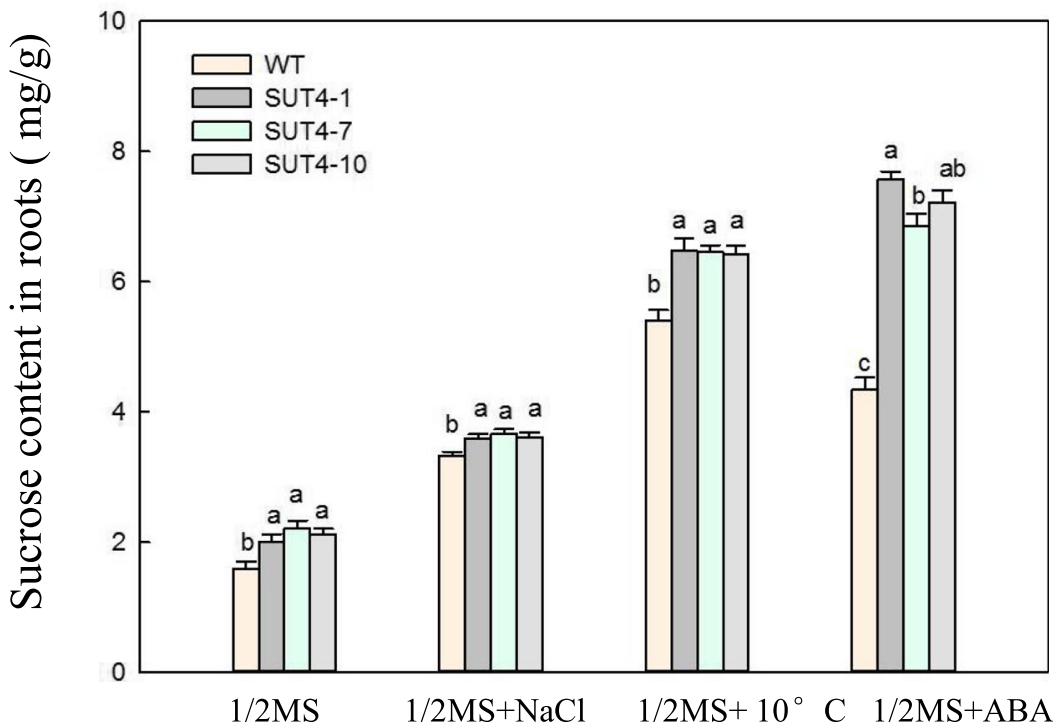

Fig. 6 The sucrose distribution was changed in IbSUT4 transgenic Arabidopsis thaliana lines under control and abiotic stress conditions, including low temperature $\left(10^{\circ} \mathrm{C}\right)$, high salt $(100 \mathrm{mM} \mathrm{NaCl})$, and exogenous $10 \mu \mathrm{M} \mathrm{ABA}$ treatments. The sucrose content of the shoots (a) and roots (b) of 14 day-old WT and transgenic seedlings under abiotic stress and ABA treatments. Lowercase letters indicate statistically significant differences $(P \leq 0.05)$

lines during stress, which may explain better transgenic plant growth under stress conditions.

Over-expression of IbSUT4 induces the expression of ABA signaling pathway genes

To explore the molecular mechanisms of the plant stress response, we obtained the 847 bp promoter of IbSUT4 by genome walking. Analyses using the plant CARE software revealed several stress and hormone response elements, including MYB, LTR, ABRE, and AuxRR-core
(Table S3). The ABRE response element was also in the IbSUT4 promoter region. The ABRE-binding transcription factors plays an important role in ABA signaling $[32,33]$, so we speculate that the over-expressed IbSUT4 lines respond to stress via ABA signaling.

We further verified the plants response to stress through the ABF-dependent ABA pathway by quantifying the expression of the ABA signaling pathway genes including ABF2, ABF4, SnRK2.2, SnRK2.3, PYL8/RCAR3 and $A B I 1$ with qRT-PCR. In the absence of ABA, the 
expression of SnRK2.2, SnRK2.3, ABF2, ABF4, and PYL8/RCAR3 did not obviously differ between the WT and three transgenic lines. Treatment with $40 \mu \mathrm{M}$ ABA for $6 \mathrm{~h}$ resulted in higher expression for most of the genes in the transgenic lines than in the WT, except $A B I 1$ (Fig. 7), a negative regulator of ABA signaling. These findings suggest that over-expression of IbSUT4 enhanced the $\mathrm{ABA}$ response, by activating the ABA receptors and positive regulators and inhibiting the negative regulator.

\section{Discussion}

Sucrose is produced in source leaves during photosynthesis and functions as a carbon source. It is transported throughout the plant by SUTs, where it regulates development and adaptation to environmental challenges. Thus, SUTs play an undeniably important role in plant growth and stress tolerance [34-37]. A more complete understanding of the mechanisms by which SUTs work can better enable the modulation of sucrose transport for better yield and improved quality in the sweetpotato. In this study, IbSUT4-a conserved SUT gene whose encoded protein is localized in the tonoplast and plasma membrane and is highly expressed in sink organs - was studied to investigate its roles in plant growth and resistance to abiotic stress. The results indicated that the over-expression of IbSUT4 in Arabidopsis thaliana might improve sucrose efflux from source leaves,

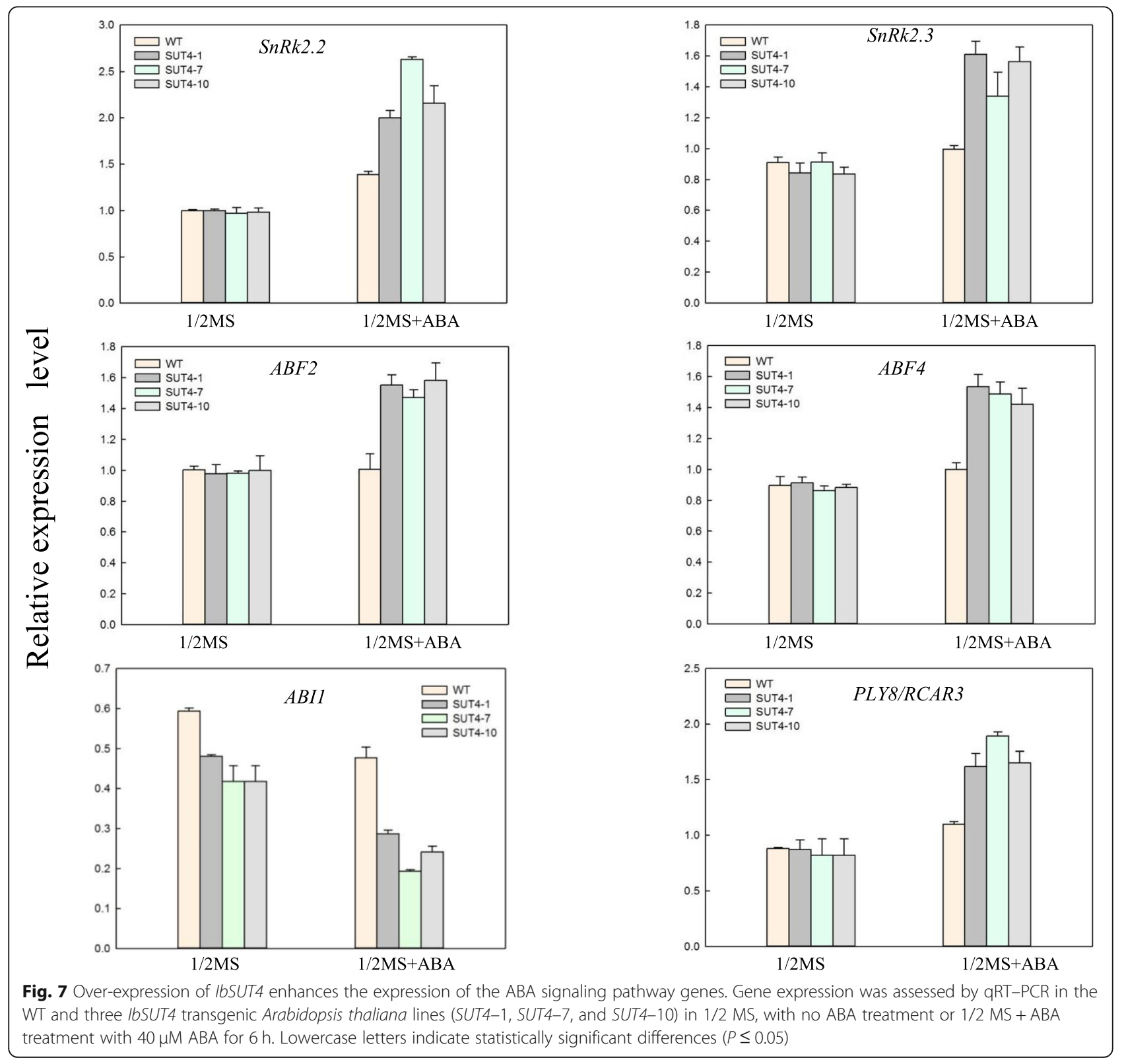


thereby increasing sucrose transport from source to sink organs, resulting in sucrose accumulation in the roots, early flowering, and improved resistance to abiotic stress through the ABF-dependent ABA signaling pathway. Our study highlights the importance of IbSUT4 in plant growth and the plant's response to abiotic stress.

\section{IbSUT4 encodes a functional protein and supports sucrose transport}

The best characterized function of the sucrose transporters in the plants is the uptake of sucrose into the phloem for long-distance photoassimilates transport. However, it has been reported that some SUTs, such as AtSUC3 and LeSUT4, can not transport sucrose [38]. A previous study showed that AtSUC6 was a pseudogenes coding for non-functional proteins [39]. More recent work has shown that AtSUC6 is a high-affinity $\mathrm{H}^{+}$- symporter mediating sucrose uptake [40]. The evolutionary distances between plants and yeast mean that the plant proteins may not target the correct membrane in yeast $[41,42]$, or that they lack the essential protein modifications and do not show normal activity in a heterologous system. Here, we used the SUSY7/Ura3 yeast strain transformed with IbSUT4 to verify sucrose uptake activity in IbSUT4. The transformed cells grew better (Fig. 3a and Fig. 3b), which was consistent with the observations of StSUT1 and AtSUT4 grown on media containing sucrose as the sole carbon source [10, 43]. Esculin is a fluorescent analog of sucrose that can be transported in plants and easily observed. Arabidopsis thaliana and tomato plants with MdSUT2 over-expression showed more intense esculin fluorescence in the root tips, indicating the sucrose uptake of MdSUT2, and suggesting enhanced activity in the MdSUT2 lines [44]. The esculin fluorescence intensity was stronger in the transgenic lines than in the WT plants (Fig. 3d). The intensity of esculin fluorescence in the transgenic Arabidopsis root tips, and their outward expansion to accumulate esculin, may verify the sucrose uptake function of IbSUT4 and explain the sequestration of esculin into the parenchymal cell vacuoles.

We present several lines of evidence to support the hypothesis that IbSUT4 mediates the source-sink distribution of sucrose and the sucrose efflux from leaves and vacuoles in the sweetpotato. IbSUT4 was localized in the tonoplast and plasma membrane (Fig. 2), where it was involved in sucrose transport across the membranes. Furthermore, the transcription level of IbSUT4 was higher in the sink tissues than in the source tissues (Fig. S1C). In dicot annuals, SUTs such as AtSUC4 are predominantly expressed in the anthers, shoot apical, and floral meristems, and the genes of the sink organs belong to clade IV $[10,45]$. This expression pattern is consistent with our observations of IbSUT4 in the root stele. AtSUC4 was verified to release sucrose from the Arabidopsis thaliana vacuoles [42]. Sinks organs are not carbon autonomous but depend on the supply of photoassimilates from the source organs. Sucrose is essential for sink metabolism and supports growth and development. Therefore, sinks retain their vacuolar sucrose at low concentrations and may use clade IV sucrose transporters to re-export sucrose. We also determined the sucrose content of the shoots and roots of WT and transgenic lines. Overexpression of IbSUT4 in Arabidopsis thaliana resulted in higher sucrose accumulation in roots than in those of the WT (Fig. 6), suggesting that IbSUT4 enhances sucrose efflux from the leaves and promote sucrose distribution to the sink organs. Payyavula et al. (2015) suggested that silencing PtaSUT4 reduced sucrose efflux from source leaves, based on observations of sucrose reduction in importing YL (young leaf) and ST (shoot tip) [12]. We hypothesized that IbSUT4 regulates the release of sucrose stored in the root vacuoles during root growth and development. When this sucrose is consumed, low cytosolic concentrations promote sucrose efflux from the source leaves. Future studies should develop transgenic IbSUT4 sweetpotato lines to verify this pathway.

\section{IbSUT4 plays important roles in plant growth and resistance to abiotic stress}

In addition to being transported from the source to the sink as the main carbohydrate, sucrose may also serve as a nonnutritional regulator of cellular metabolism by altering gene expression [46]. Therefore, SUTs play a vital role in plant growth and the response to abiotic stress. In higher plants, flowering signifies the transition from the vegetative to the reproductive phase and is important for plant growth and development. It is controlled by many transduction pathways, including the photoperiod-dependent phytochrome signal transduction pathway, the sucrose pathway, and the $\mathrm{GA}_{3}$ pathway [47-49]. FT encodes a protein similar to the phosphatidylethanolamine-binding protein and promotes flowering under LD conditions [50,51]. In this study, we observed early flowering and increased AtFT expression in the IbSUT4 expressing lines (Fig. 4). This suggests that IbSUT4 is involved in plant growth and development.

Previous studies have shown significantly lower MDA content in three MdSUT2 over-expression lines (MdSUT2/Col-1/2/3) under stress treatment, indicating that MdSUT2 plays important roles in resistance to abiotic stress, and this was further supported by the root lengths analysis [44]. The rate of the superoxide anion production increases with improved stress intensity, causing an imbalance in the metabolism of superoxide anions in the cell. This results in excess superoxide anions that induce a free radical chain reaction, generating more free radicals and reactive oxygen species, which oxidizes the double bonds of unsaturated fatty acids in 
the membrane cells, leading to break down and destruction. MDA is an important product of lipid peroxidation in the cell membranes, whose concentration varies in response to biotic and abiotic stressors [52]. Here, we found that less MDA was generated in the IbSUT4 transgenic Arabidopsis thaliana seedlings (Fig. 5d), indicating that over-expression of IbSUT4 can significantly improve plant tolerance to stress conditions.

Generally, environmental stress affects sucrose supply by inhibiting photosynthesis. Therefore, the trans-membrane transport and distribution of sucrose in plants is a key step in plant stress resistance. To date, the cumulative evidence suggests that SUTs influence the plant stress response by altering the sucrose allocation from the source to sink organs. PtaSUT4-RNAi caused substantial sucrose accumulation in the vacuole and affected the water balance to such an extreme that PtaSUT4-RNAi plants wilt in short-term drought conditions [14]. Loss-of-function mutation in AtSUC2 and AtSUC4 led to hypersensitive responses to abiotic stress and ABA treatment during seed germination and seedling growth and higher sucrose levels in the shoots [13]. High sucrose content in the shoots and low sucrose content in the roots were observed in the AtSUC4 mutant, under drought and salt stress [53]. The over-expression of IbSUT4 caused a greater accumulation of sucrose in the roots and a lower accumulation in the shoots, under abiotic stress conditions (Fig. 6). The transgenic IbSUT4 lines responded to abiotic stress by altering their sucrose allocation, highlighting the potential of IbSUT4 in the stress response.

\section{IbSUT4 responds to abiotic stress through the ABF- dependent $A B A$ signaling pathway}

The plant hormone ABA is involved in many processes of plant growth and development, including inhibiting seed germination, maintaining seed dormancy, controlling stomatal closure, and the adaptive responses to environmental stress [54, 55]. Adaptation to abiotic stress is regulated by $\mathrm{ABA}$-dependent and $\mathrm{ABA}$-independent pathways [56, 57]. Disruption of AtSUC2, AtSUC4, and AtSUC9 results in hypersensitive responses to abiotic stress, and $A B A$ treatment during seed germination and seedling growth inhibits the expression of ABA-induced and ABA-responsive genes [13, 23], suggesting that AtSUC2, AtSUC4, and AtSUC9 are involved in the abiotic stress response through the ABA-dependent signaling pathway. The ABA-dependent signaling pathway in plants operates by changing the expression of ABAregulated genes containing $A B F s, S N F 1$, and $P P 2 C$, such as $A B I 1, A B I 2$ [18, 58-60]. Here, our promoter analyses confirmed the presence of the ABRE-motif in the IbSUT4 promoter (Table S3). ABRE is a conserved ABA-responsive cis-element that controls ABA-regulated gene expression $[18,61]$. MdAREB2 combined with the ABRE-motif element in the MdSUT2 promoter regulated the stressinduced response of sucrose accumulation via ABAsignaling pathway [33]. Further analyses of the ABAregulated gene expression profiles showed that $A B F s$ $(A B F 2, A B F 4), A B F s$ upstream (SnRK2.2, SnRK2.3), and ABA receptor (PYL8/RCAR3) were morer highly expressed in the $\mathrm{ABA}$ treatment, except $\mathrm{ABF}$ downstream (ABI1) (Fig. 7). Several instances of cross-talk between the stress and ABA signaling pathways involved SnRKs (sucrose nonfermenting-related protein kinase), which are key metabolic regulators of the stress response [61]. Over-expression of SnRK2C in Arabidopsis thaliana improved the expression of stress response genes during drought tolerance [19]. $A B I 1$ (ABA insensitive 1), a member of the PP2C gene family, is a negative regulator of $A B A$ signal transduction. $B c A B I 1$ is a highly homologous gene to AtABI1 that presents a constitutive expression pattern and participates in wound stress and high salt responses [62]. Thus, we speculate that IbAREBs combine with the ABRE-motif in the IbSUT4 promoter to respond to stress through the ABFdependent ABA signaling pathway.

\section{Conclusions}

In summary, IbSUT4 is an important SUT gene, encoding a protein that is localized in the tonoplast and plasma membrane. It also influences the sucrose distribution between source and sink tissues. Over-expression of this gene accelerated Arabidopsis thaliana growth and improved abiotic stress resistance by enhancing the sucrose content of the sink organs. Our study reveals the critical role of IbSUT4 in plant growth and abiotic stress response. The function of SUTs is highly conserved, so the accumulation of sucrose in Arabidopsis roots suggests that IbSUT4 influences the quality and yield of root crops, especially sweetpotato (Fig. 8). Future studies should verify these functions by developing transgenic sweetpotato lines. A more complete understanding of how IbSUT4 affects the sucrose distribution of plants may open new avenues for improving crop yield.

\section{Methods}

\section{Plant materials and growth conditions}

These Arabidopsis thaliana and tobacco plants used in this study were obtained in a climate chamber at the Institute of Plant Physiology and Ecology, Chinese Academy of Sciences, Shanghai $\left(31^{\circ} 18^{\prime} \mathrm{N}, 121^{\circ} 43^{\prime} \mathrm{E}\right)$. The Ipomoea batatas "Taizhong 6" was cultivated in the field at the Wushe Plantation for Transgenic Crops, Shanghai $\left(31^{\circ} 14^{\prime} \mathrm{N}, 121^{\circ} 29^{\prime} \mathrm{E}\right)$.

Arabidopsis thaliana (Columbia ecotype, Col-0), used for experiments, were maintained under LD conditions, at approximately $600 \mathrm{mmolm}^{-2} \mathrm{~s}^{-1}$, at $25^{\circ} \mathrm{C}$. The seed germination experiments and stress tolerance assays 


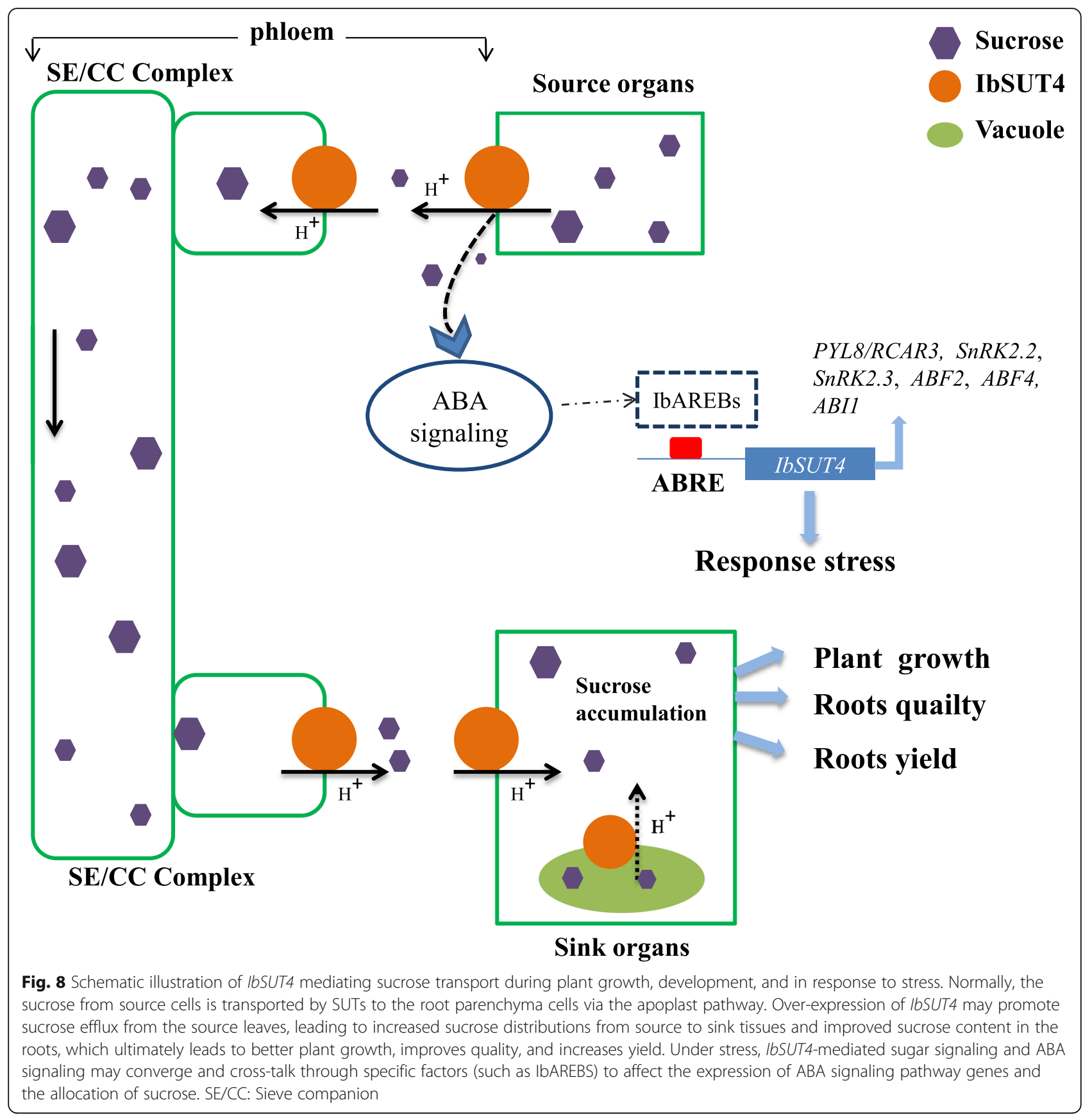

were performed according to the method described by Mas [44], with some modifications. We added a $10^{\circ} \mathrm{C}$ processing. For the plant phenotype analyses, WT and transgenic plants were germinated on the MS medium for $10 \mathrm{~d}$ and then grown in individual pots in a greenhouse under LD conditions, with 6 replicates. For the analyses of the ABA signaling pathway genes, WT and transgenic plants were grown on $1 / 2 \mathrm{MS}$ medium for 14 $\mathrm{d}$, and then treated with $40 \mu \mathrm{M} \mathrm{ABA}$ for $6 \mathrm{~h}$.

The tobacco (Nicotiana benthamiana Domin; all in this lab) used for the subcellular localization assays were grown in the greenhouse, in potting soil, and under LD conditions.

Sweetpotato (Ipomoea batatas (L.) Lam; all in this lab) "TaiZhong 6", used for gene expression analyses, was grown in Shanghai, in a Wushe farm. Tissues were sampled from 3 month-old sweetpotato plants.

\section{Cloning of the sucrose transporter cDNAs}

Approximately $100 \mathrm{mg}$ of sweetpotato source leaves were ground with liquid nitrogen and transferred to a $1.5 \mathrm{~mL}$ centrifuge tube. Total RNAs were extracted from 
the source leaves using RNAprep Pure Plant Kit (TIANGEN, China). E4-F and E4-R primers (Table S1) were designed according to the conserved sequences of plant SUT genes, and the EST fragment of IbSUT4 gene was amplified and sequenced.

To acquire the full-length SUT cDNA sequences, two rounds of nested rapid amplification of cDNA ends (RACE) PCR techniques were performed. SpSUT4F/R primers (Table S1) were designed according to the EST sequences, and the $5^{\prime}$ and $3^{\prime}$ ends of IbSUT4 cDNA were amplified and sequenced, using SMART RACE cDNA Amplification Kit (Clontech, Japan), according to the manufacturer's instructions. The full-length IbSUT4 sequence was obtained by splicing sequences, and the IbSUT4-F/R primers (Table S1) were designed according to the full-length sequences, which was then amplified using cDNA as the template and ReverTra Ace qPCR RT Master Mix's (TOYOBO, Japan), according to the manufacturer's instructions.

\section{Sequence alignment and phylogenetic tree construction}

To identify the homologs of IbSUT4, BlastP searches using the full-length IbSUT4 protein sequences were performed against the sequences of Arabidopsis thaliana, Nicotiana tabacum, Solanum tuberosum, Solanum lycopersicum, Malus domestica, in the National Center for Biotechnology Information (NCBI). Protein sequences were aligned using MEGA5.2, and unrooted phylogenetic trees were constructed using the NeighborJoining method in MEGA5.2. The reliability of the trees was estimated with 1000 bootstrap iterations.

Subcellular localization analyses and esculin uptake assay To determine the subcellular localization of IbSUT4 in sweetpotato, ORFs, without the stop codon, were amplified by PCR (Table S1). The IbSUT4 amplified product was digested with SalI/SpeI and then cloned into the region between the CaMV35S promoter and GFP of the PUC18 binary vector. The resulting CaMV35S:IbSUT4GFP (PUC18) construct was used to transform tobacco protoplasts. Leaf mesophyll protoplasts from twomonth-old tobacco plants were generated as described by Drechsels [63]. After transformation via the polyethylene glycol method [64]. Protoplasts were kept in tubes for approximately $18 \mathrm{~h}$ at $22^{\circ} \mathrm{C}$ in darkness. PI in W5 buffer was added to $500 \mu \mathrm{L}$ of protoplasts suspension in W5 solution $(\mathrm{pH}=5.7)$ in a $1.5 \mathrm{~mL}$ tube to a final concentration of $50 \mu \mathrm{g} \mathrm{mL}^{-1}$. The tubes were gently inverted, and the protoplasts were incubated for $30 \mathrm{~min}$ at room temperature in darkness. The solution was centrifuged and washed with W5 solution after discarding the supernatant.

Esculin uptake by the seedlings was assessed according to the methods described by Ma QiJuns [44]. Quantitative analyses of the fluorescence intensity were conducted in Image J software by calculating the average optical density; the value of IntDen / Area of three pictures.

Protoplasts and Arabidopsis thaliana were observed using an Olympus FV1000 confocal laser-scanning microscope, equipped with a 603/1.2 NA UPLSAPO oil immersion objective lens (Olympus). GFP fluorescence was observed using excitation and emission wavelengths of $473 \mathrm{~nm}$ and $487-521 \mathrm{~nm}$, respectively. Chloroplast autofluorescence was observed by excitation and emission at $559 \mathrm{~nm}$ and $606-673 \mathrm{~nm}$, respectively. PI fluorescence was observed by excitation and emission at $535 \mathrm{~nm}$ and $615 \mathrm{~nm}$, respectively. Esculin fluorescence was observed by excitation and emission at $367 \mathrm{~nm}$ and $454 \mathrm{~nm}$, respectively.

\section{Functional analysis of IbSUT4 in yeast SUSY7/ura3}

The yeast mutant SUSY7/ura3 can not synthesize invertase [4]. It can metabolize sucrose in the cell and grow normally on the media containing sucrose as the sole carbon source, when the gene encoding the sucrose synthase in the plant is tranferred into SUSY7/ura3. Heterologous expression and complementation of sucrose in the defective yeast is direct envidence to verify the sucrose transport activity of the SUT genes. We cloned the IbSUT4 ORF by PCR (Table S1). The IbSUT4 ORF was excised with ClaI/SmaI, and then cloned into the same sites of p416GPD. To analyze the SUT activity, the vector (IbSUT4-P416) was transferred into SUSY7/ura3 competent cells. Large, healthy colonies from the bait and control strains were selected and resuspend individually in $0.9 \% \mathrm{NaCl}$, and the OD600 was adjusted to 1. Each transformation reaction was then diluted 10 , 100,1000 , and 10,000 times. Ten microliters of each dilution reaction were plated on an agar medium containing $1.7 \mathrm{~g} \mathrm{~L}^{-1}$ yeast nitrogen base without amino acids (Difco), $2 \%$ sucrose (Difco) or $2 \%$ glucose (Difco), $5 \mathrm{~g}$ $\mathrm{L}^{-1}$ ammonium sulfate, $20 \mathrm{mg} \mathrm{L}^{-1}$ tryptophan, and $1.5 \%$ agarose, and the $\mathrm{pH}$ was adjusted to 5.0 using $\mathrm{HCl}$. The plates were maintained at $30^{\circ} \mathrm{C}$ for $3 \mathrm{~d}$ and then observed and photographed.

\section{Amplification of the IbSUT4 promoter}

To analyze the promoter of the IbSUT4, we amplified the promoter by genome walking method. Primers were designed from known DNA sequences in the inverse direction. The reverse primer was designed from the $5^{\prime}$ end, and the forward primer was designed from the 3' end. Primers were located at 100-200 bp away from the ends (Table S1). First, $5 \mu \mathrm{g}$ genomic DNA was digested with a restriction enzyme that does not uncut between the primers. The digestion reaction was carried out at $37^{\circ} \mathrm{C}$ for $6-8 \mathrm{~h}$. The digested DNA was purified with an equal volume of phenol/chloroform, centrifuged at 10 , 
$000 \mathrm{~g}$ at $4{ }^{\circ} \mathrm{C}$ for $10 \mathrm{~min}$, and the upper phase was collected. An equal volume of chloroform/isoamyl alcohol mixture was further added, the solution was vortexed and centrifuged, and the upper phase was collected. The DNA was precipitated with 0.1 volume $3 \mathrm{M}$ sodium acetate and 2.5 volume $100 \%$ ethanol and centrifuged, and the supernatant was discarded. The pellet was washed with $80 \%$ ethanol and centrifuged, and the supernatant was discarded. The pellet was air-dried and then resuspended in water, and the T4 enzyme was used for ligation. These steps were repeated to precipitate and depurated the DNA, and two rounds of PCR were performed to amplify the promoter.

\section{Transformation of IbSUT4 into Arabidopsis}

The full-length coding sequence of IbSUT4 was amplified by PCR and inserted into the pCAMBIA1301 vector. The resulting 35S:IbSUT4 construct was introduced into the Agrobacterium strain GV3101 for Arabidopsis thaliana transformation using the floral dip method [65]. The seeds were collected and sown on $1 / 2$ MS medium containing $25 \mathrm{mg} \mathrm{L}^{-1}$ hygromycin for selection.

\section{qRT-PCR assays}

Gene expression was quantified with specific qRT-F and qRT-R primers. Actin was used as the control. The primers used for assays are shown in Table S1. Each sample was assayed in three biological replicates. The relative quantification of specific mRNA levels was performed using the cycle threshold (Ct) $2^{-\mathrm{DDCt}}$ method [66].

\section{Determination of sucrose content and malondialdehyde (MDA) content}

The sucrose concentrations were determined by highperformance liquid chromatography (HPLC), as described by Gongs [13].

The MDA content was determined according to the method described by Mas [44], with some modifications. We used 10\% TCA to extract MDA from the roots.

\section{Supplementary information}

Supplementary information accompanies this paper at https://doi.org/10. 1186/s12870-020-02382-8.

Additional file 1: Table S1. Sequences of the primers used in this study Table S2. Count the number of flowering plants. Table S3. Ciselement of the IbSUT4 promoter. Figure S1. A. EST sequences. B. The 5'RACE and 3'-RACE amplification of IbSUT4. C. Expression of IbSUT4 gene was assessed by qRT-PCR in the different tissues of "Taizhong 6" sweetpotato. Three-month-old samples were collected from the field. DL: development leaf, B: handle, S: stem, WR: white root, RR: red root, DR: development root, MR: mature root. (A, B) The gels are cropped from Additional file 2, Figure S.1. Lowercase letters indicate statistically significant differences $(P \leq 0.05)$. Figure S2. In vivo sucrose uptake activity of IbSUT4. The average optical density was significantly higher in the roots of transgenic plants than in the WT plants. Average optical density = IntDen/Area. Lowercase letters indicate statistically significant differences
$(P \leq 0.05)$. Figure S3. The expression of IbSUT4 in the roots and leaves of one-month-old plant treated with low temperature $\left(4^{\circ} \mathrm{C}\right)$, high salt (200 $\mathrm{mM} \mathrm{NaCl})$, drought stress (300 mM Mannitol), or exogenous ABA (25 $\mu \mathrm{M})$. A. Quantification of IbSUT4 expression in the leaves. B. Quantification of IbSUT4 expression in the roots. Lowercase letters indicate statistically significant differences $(P \leq 0.05)$.

Additional file 2 Figure S1. The original and full-length gels of EST, 5'RACE and 3'-RACE. A. EST4 sequences. B. The 5'-RACE and 3'-RACE amplification of IbSUT4. Figure S2. The original and full-length gel of RT-PCR analysis of IbSUT4 expression in the WT and three transgenic lines. Figure S3. The original blot of IbSUT4 function analysis in the SUSY7/Ura3 yeast strain. A. SUSY7/ura3 yeast transformed with the empty P416 vector and with IbSUT4 in the P416 vector, and grown on 2\% sucrose. B. SUSY7/ura3 yeast transformed with the empty P416 vector and with IbSUT4 in the P416 vector, and grown on $2 \%$ glucose.

\section{Abbreviations}

ABA: Abscisic acid; ABFs: ABA-responsive element binding factors; B: Handle; DL: Development leaf; DR: Development root; HPLC: High-performance liquid chromatography; LD: Long Day (16 h light / 8 h dark);

MDA: Malondialdehyde; MFS: Major Facilitator Superfamily; MR: Mature root; MS: Murashige and Skoog; NCBI: National Center for Biotechnology Information; ORF: Open reading frame; PI: Propidium lodide; qRTPCR: Quantitative reverse- transcription-PCR; RACE: Rapid Amplification of cDNA Ends; RR: Red root; S: Stem; SE-CCs: Sieve element-companion cells; ST: Shoot tip; SUTS or SUCs: Sucrose transporters or carriers;

TCA: Trichloroacetic acid; WT: Wild-type; WR: White root; YL: Young leaf

\section{Acknowledgments}

We thank Wu Xiaoyun and Zhang Yandi (Unniversity of Chinese Academy of Sciences) for their advice assistance. We would like to thank Editage (www. editage.cn) for English language editing.

\section{Authors' contributions}

WDD planed and performed the experiments and prepared the manuscript. LHJ edited the manuscript and gave advices regarding the work. WHX and ZP gave advices regarding the work and provides a place in which do experiments. SCY supervised the work and gave interpretation of results. All authors have read and approved the manuscript

\section{Funding}

This work was supported by National Natural Science Foundation of China Youth Fund (Grant No. 31701357) and Shandong Agriculture Innovation Team (Grant No.SDAIT-16-01). These founding sponsors had no role in the design of the study; in the collection, analyses, or interpretation of data; in the writing of the manuscript, and in the decision to publish the results.

\section{Availability of data and materials}

The datasets supporting the conclusions of this article are included within the article and its additional files. About genes database could download from NCBI by their accession number. The accession numbers of these genes are as follows: AtFT (At1g65480), AtABI3(At3g24650), ABF2 (ABO17160), ABF4 (At3g19290), SnRK2.2 (AEE78673), SnRK2.3 (NP201489), PYL8/RCAR3 (At5g53160) and ABI1 (At4g26080), IbSUT1 (ADK62516.1), IbSUT2 (ADL63117.1), IbSUT3 (MN233361), IbSUT4 (MN233360), LeSUT1 (CAA57726.1), LeSUT2 (AAG12987.1), LeSUT4 (AAG09270.1), StSUT1 (CAA48915.1), StSUT2

(AAP43631.1), StSUT4 (AAG25923.2), NtSUT1 (CAQ58421.1), NtSUT3

(AAD34610.1), NtSUT4 (BAl60050.1), AtSUC1 (AAV97807.1), AtSUC2

(AAN31829.1), AtSUC4 (AAO42412.1), AtSUC5 (NP177334.1), AtSUC7 (NP001031242.1), AtSUC8 (AEC06318.1), AtSUC9 (NP196235.1), MdSUT4 (NP001280809).

Ethics approval and consent to participate

Not applicable.

\section{Consent for publication}

Not applicable.

\section{Competing interests}

The authors declare that they have no competing interest. 


\section{Author details}

'State Key Laboratory of Crop Biology, College of Agronomic Science, Shandong Agricultural University, Tai' an 271018, China. ${ }^{2}$ National Key of Plant Molecular Genetics, CAS Center for Excellence in Molecular Plant Sciences, Institute of Plant Physiology and Ecology, Chinese Academy of sciences, Shanghai 200032, China.

\section{Received: 27 December 2019 Accepted: 2 April 2020}

\section{Published online: 15 April 2020}

\section{References}

1. Lemoine R, La Camera S, Atanassova R, Dédaldéchamp F, Allario T, Pourtau $\mathrm{N}$, et al. Source to sink transport and regulation by environmental factors. Front Plant Sci. 2013;4:272.

2. Yan N. Structural advances for the major facilitator superfamily (MFS) transporters. Trends Biochem Sci. 2013;38(3):151-9.

3. Williams LE, Lemoine R, Sauer N. Sugar transporters in higher plants: a diversity of roles and complex regulation. Trends Plant Sci. 2000;5:283-90.

4. Riesmeier JW, Willmitzer L, Frommer WB. Isolation and characterization of a sucrose carrier cDNA from spinach by functional expression in yeast. EMBO J. 1992;11(13):4705-13.

5. Sauer N, Stolz J. SUC1 and SUC2: two sucrose transporters from Arabidopsis thaliana: expression and characterization in baker's yeast and identification of the histidine-tagged protei. Plant J. 1994;6:67-77.

6. Gould N, Thorpe MR, Pritchard J, et al. AtSUC2 has a role for sucrose retrieval along the phloem pathway: evidence from carbon-11 tracer studies. Plant Sci. 2012;188-189:97-101.

7. Srivastava AC, Ganesan S, Ismail IO, et al. Functional characterization of the Arabidopsis AtSUC2 sucrose/ $\mathrm{H}^{+}$symporter by tissue-specific complementation reveals an essential role in phloem loading but not in long-distance transport. Plant Physiol. 2008;148:200-11.

8. Gottwald JR, Krysan PJ, Young JC, et al. Genetic evidence for the in planta role of phloem-specific plasma membrane sucrose transporters. Proc Natl Acad Sci. 2001;97:13979-84.

9. Sivitz AB, Reinders A, Johnson ME, et al. Arabidopsis sucrose transporter AtSUC9 high-affinity transport activity, intragenic control of expression, and early flowering mutant phenotype. Plant Physiol. 2006;143(1):188-98.

10. Weise A. A new subfamily of sucrose transporters, SUT4, with low affinity/ high capacity localized in enucleate sieve elements of plants. Plant Cell. 2000;12(8):1345-56.

11. Chincinska IA, Liesche J, Krügel U, et al. Sucrose transporter StSUT4 from potato affects flowering, tuberization, and shade avoidance response. Plant Physiol. 2008;146(2):515-28.

12. Payyavula RS, Tay KH, Tsai CJ, et al. The sucrose transporter family in Populus: the importance of a tonoplast PtaSUT4 to biomass and carbon partitioning. Plant J. 2015:65(5):757-70.

13. Gong X, Liu M, Zhang L, et al. Arabidopsis AtSUC2 and AtSUC4, encoding sucrose transporters, are required for abiotic stress tolerance in an ABAdependent pathway. Physiol Plant. 2014;153(1):119-36.

14. Frost CJ, Nyamdari B, Tsai CJ, et al. The Tonoplast-localized sucrose transporter in Populus (PtaSUT4) regulates whole-plant water relations, responses to water stress, and photosynthesis. PLoS One. 2012;7(8):e44467.

15. Xu Q, Chen S, Yun JR. Regulation of sucrose transporters and phloem loading in response to environmental cues. Plant Physiol. 2018;176:930-45.

16. Raghavendra AS, Gonugunta VK, Christmann A, et al. ABA perception and signalling. Trends in Plant Sci. 2010;15(7):395-401.

17. Sussmilch FC, Atallah NM, Brodribb TJ, et al. Abscisic acid (ABA) and key proteins in its perception and signalling pathways are ancient, but their roles have changed through time. Plant Signaling Behav. 2017:12(9): e1365210.

18. Choi $\mathrm{HI}$, Hong JH, Ha JO, et al. ABFs, a family of ABA-responsive element binding factors. J Biol Chem. 2000;275(3):1723-30.

19. Umezawa T, Yoshida R, Maruyama K, et al. SRK2C, a SNF1-related protein kinase 2, improves drought tolerance by controlling stress-responsive gene expression in Arabidopsis thaliana. Proc Natl Acad Sci. 2004;101:17306-11.

20. Dong T, Park Y. Abscisic acid: biosynthesis, inactivation, homoeostasis and signalling. Essays Biochem. 2015;58:29-48.

21. Wang Y, Chen P, Sun L, et al. Transcriptional regulation of PaPYLS, PaPP2CS and PaSnRK2s during sweet cherry fruit development and in response to abscisic acid and auxin at onset of fruit ripening. Plant Growth Regul. 2015; 75(2):455-64.
22. Rook F, Corke F, Card R, et al. Impaired sucrose-induction mutants reveal the modulation of sugar-induced starch biosynthetic gene expression by abscisic acid signaling. Plant J. 2001;26:421-33.

23. Jia W, Zhang L, Wu D, et al. Sucrose transporter AtSUC9 mediated by a low sucrose level is involved in Arabidopsis abiotic stress resistance by regulating sucrose distribution and ABA accumulation. Plant Cell Physiol. 2015;56(8):1574.

24. Lalonde $S$, Wipf $D$, Frommer WB. Transport mechanisms for organic forms of carbon and nitrogen between source and sink. Annu. Rev. Plant Biol. 2004;55:341-72.

25. Li Y, Gu Y, Qin H, et al. Two pairs of sucrose transporters in Ipomoea batatas (L.) lam are predominantly expressed in sink leaves and source leaves respectively. Plant Sci 2010; 179(3): \-256.

26. Kühn C, Grof CP. Sucrose transporters of higher plants. Curr Opin Plant Biol. 2010;13:287-97.

27. Chincinska I, Gier K, Krügel, Undine, et al. Photoperiodic regulation of the sucrose transporter StSUT4 affects the expression of circadian-regulated genes and ethylene production. Front. Plant Sci. 2013;4:26.

28. Cheng XF, Wang ZY. Overexpression of COL9, a CONSTANS-LIKE gene, delays flowering by reducing expression of $\mathrm{CO}$ and $\mathrm{FT}$ in Arabidopsis thaliana. Plant J. 2010;43(5):758-68.

29. Yamaguchi S, Kamiya Y. Gibberellins and light-stimulated seed germination. J Plant Growth Regul. 2001;20(4):369-76.

30. Sariyildiz Z, Demir I, Halloran N. Germination at stress temperatures and ABA content of Pepper (Capsicum annuum L.) seeds in relation to seed development. Eur J Hortic Sci. 2005;70(2):67-70.

31. Barros Galvão, Thiago. Regulation of seed dormancy and germination in Arabidopsis thaliana. Ph.D. thesis, Dissertation of University of York, White Rose eTheses Online(etheses.whiterose.ac.uk). 2017.

32. Fujita Y, Fujita AM, Satoh CR, et al. AREB1 is a transcription activator of nove ABRE-Dependent ABA signaling that enhances drought stress tolerance in Arabidopsis. Plant Cell. 2005;17(12):3470-88.

33. Ma QJ, Sun MH, Liu YJ, et al. Transcription factor AREB2 is involved in soluble sugar accumulation by activating sugar transporter and amylase genes. Plant Physiol. 2017;174:2348.

34. Noiraud N, Delrot S, Lemoine R. The sucrose transporter of celery identification and expression during salt stress. Plant Physiol. 2000; 122(4):1447-55.

35. Ramon M, Rolland F, Sheen J. Sugar sensing and signaling. Arabidopsis Book. 2008;6:e0117.

36. Lemoine R, La CS, Atanassova R, et al. Source-to-sink transport of sugar and regulation by environmental factors. Front Plant Sci. 2013;4:272.

37. Krügel, Undine, Kühn, Christina. Post-translational regulation of sucrose transporters by direct protein-protein interactions. Front Plant Sci. 2013;4:237.

38. Peng $D, G u X, X u e ~ L$, et al. Bayesian phylogeny of sucrose transporters: ancient origins, differential expansion and convergent evolution in monocots and dicots. Front. Plant Sci. 2014;5:615.

39. Sauer N, Ludwig A, Knoblauch A, Rothe P, Gahrtz M, Klebl F. AtSUC8 and AtSUC9 encode functional sucrose transporters, but the closely related AtSUC6 and AtSUC7 genes encode aberrant proteins in different Arabidopsis ecotypes. Plant J. 2004;40:120-30.

40. Rottmann TM, Fritz C, Lauter A, et al. Protoplast-esculin assay as a new method to assay plant sucrose transporters: characterization of AtSUC6 and AtSUC7 sucrose uptake activity in Arabidopsis Col-0 Ecotype. Front Plant Sci. 2018;9:430.

41. Barker L, Kühn C, Weise A, Schulz A, Gebhardt C, Hirner B, et al. SUT2, a putative sucrose sensor in sieve elements. Plant Cell. 2000;12:1153-64.

42. Schneider S, Hulpke S, Schulz A, Yaron I, Höll J, Imlau A, et al. Vacuoles release sucrose via tonoplast-localised SUC4-type transporters. Plant Biol. 2012:24:325-36

43. Riesmeier JW, Willmitzer L, Frommer WB. Antisense repression of the sucrose transporter affects assimilate partitioning in transgenic potato plants. EMBO J. 1994;3:1-7.

44. Ma QJ, Sun MH, Liu YJ, et al. Molecular cloning and functional characterization of the apple sucrose transporter gene MdSUT2. Plant Physiol Biochem. 2016;109:442-51.

45. Endler A, Meyer S, Schelbert S, et al. Identification of a vacuolar sucrose transporter in barley and Arabidopsis mesophyll cells by a tonoplast proteomic approach. Plant Physiol. 2006;141:196-207.

46. Rolland $F$, Baenagonzalez $E$, Sheen J. Sugar sensing and signaling in plants: conserved and novel mechanisms. Annu Rev Plant Biol. 2006;57: 675-709. 
47. Kojima S, Takahashi Y, Kobayashi Y, et al. Hd3a, a rice ortholog of the Arabidopsis FT gene, promotes transition to flowering downstream of Hd1 under short-day conditions. Plant Cell Physiol. 2002;43(10):1096-105.

48. Searle I, Coupland G. Induction of flowering by seasonal changes in photoperiod. EMBO J. 2002;23(6):1217-222.

49. Kardailsky I. Activation tagging of the floral inducer. Science. 1999;286: $1962-5$.

50. Kobayashi Y. A Pair of related genes with antagonistic roles in mediating flowering signals. Science. 1999;286:1960-2.

51. Gong X, Liu ML, Wang C, et al. Sucrose transporter gene AtSUC4 regulates sucrose distribution and metabolism in response to salt stress in Arabidopsis thaliana. Advanced Materials Research. 2013;726-731:217-21.

52. Davey MW, Stals E, Panis B, et al. High-throughput determination of malondialdehyde in plant tissues. Analytical Biochemistry. 2005;347(2):201-7.

53. Gong X, Liu ML, Zhang $L$, et al. Sucrose transporter gene AtSUC4 responds to drought stress by regulating the sucrose distribution and metabolism in Arabidopsis thaliana. Adv Mater Res. 2013:971-2975.

54. Finkelstein RR, Gampala SSL, Rock CD. Abscisic acid signaling in seeds and seedlings. Plant Cell. 2002;14:S15.

55. Desikan $\mathrm{R}$, Cheung MK, Bright J, et al. ABA, hydrogen peroxide and nitric oxide signalling in stomatal guard cells. J Exp Bot. 2004;55(395):205-12.

56. Shinozaki K, Yamaguchi-shinozaki K, Seki M. Regulatory network of gene expression in the drought and cold stress responses. Curr Opin Plant Biol. 2003;6(5):410-17.

57. Nakashima K, Yamaguchishinozaki K, Shinozaki K. The transcriptional regulatory network in the drought response and its crosstalk in abiotic stress responses including drought, cold, and heat. Front Plant Sci. 2014;5: 170.

58. Ma Y, Szostkiewicz I, Korte A, et al. Regulators of PP2C phosphatase activity function as Abscisic Acid Sensors. Science. 2009;324:1172408.

59. Abdeen A, Schnell J, Miki B. Transcriptome analysis reveals absence of unintended effects in drought-tolerant transgenic plants overexpressing the transcription factor ABF3. Bmc Genomics. 2010;11:69.

60. Busk PK. Montserrat Pagès, Regulation of abscisic acid-induced transcription. Plant Mol Biol. 1998;37:425-35.

61. Hey SJ, Byrne E, Halford NG.The interface between metabolic and stress signaling. Ann Bot. 2010;105(2):197-203.

62. Kong L, Hang D, Shuai H, et al. Isolation, expression, and evolution analysis of the type $2 \mathrm{C}$ protein phosphatase gene $B C A B / 1$ involved in abiotic and biotic stress in Brassica campestris ssp. Chinensis. Plant Growth Regul. 2018; 85:1-11.

63. Drechsel G, Bergler J, Wippel K, Sauer N, Vogelmann K, and Hoth S. Cterminal armadillo repeats are essential and sufficient for association of the plant U-box armadillo E3 ubiquitin ligase SAUL1 with the plasma membrane. Exp Bot. 2011;62:775-85.

64. Abel S, Theologis A. Transient transformation of Arabidopsis leaf protoplasts: a versatile experimental system to study gene expression. Plant J. 1994;5: 421-27.

65. Clough SJ. Floral Dip: Agrobacterium-mediated germ line transformation. Methods Mol Biol. 2005;286:91-102.

66. Livak K, Schmittgen TD. Analysis of relative gene expression data using real-time quantitative PCR and the 2(-Delta Delta C(T)) Method. Methods. 2001;25:402-8

\section{Publisher's Note}

Springer Nature remains neutral with regard to jurisdictional claims in published maps and institutional affiliations.

Ready to submit your research? Choose BMC and benefit from:

- fast, convenient online submission

- thorough peer review by experienced researchers in your field

- rapid publication on acceptance

- support for research data, including large and complex data types

- gold Open Access which fosters wider collaboration and increased citations

- maximum visibility for your research: over $100 \mathrm{M}$ website views per year

At BMC, research is always in progress.

Learn more biomedcentral.com/submissions 